\title{
BMJ Open Process evaluation of the central chronic medicines dispensing and distribution programme in Namakwa district, Northern Cape province protocol: a multimethod approach
}

\author{
Kim Grace Smith, ${ }^{1}$ Edward Nicol (1) ${ }^{1,2}$
}

To cite: Smith KG, Nicol E. Process evaluation of the central chronic medicines dispensing and distribution programme in Namakwa district, Northern Cape province protocol: a multimethod approach. BMJ Open 2020;10:e032530. doi:10.1136/ bmjopen-2019-032530

- Prepublication history and additional material for this paper are available online. To view these files, please visit the journal online (http://dx.doi. org/10.1136/bmjopen-2019032530).

Received 26 June 2019 Revised 02 December 2019 Accepted 17 December 2019

Check for updates

(c) Author(s) (or their employer(s)) 2020. Re-use permitted under CC BY-NC. No commercial re-use. See rights and permissions. Published by BMJ.

${ }^{1}$ Division of Health Systems and Public Health, Global Health Department, Stellenbosch University, Cape Town, Western Cape, South Africa

${ }^{2}$ Burden of Disease Research Unit, South African Medical Research Council, Cape Town, Western Cape, South Africa

Correspondence to

Dr Edward Nicol;

Edward.Nicol@mrc.ac.za

\section{ABSTRACT}

Introduction The quadruple burden of disease in South Africa, including the HIV/AIDS epidemic, has placed enormous strains on public healthcare (PHC) facilities. These strains specifically compromised the resources available to deal with high volumes of chronic diseases that contribute to medicine shortages and poor service delivery. In an attempt to address these challenges, the Central Chronic Medicines Dispensing and Distribution (CCMDD) programme, which aimed to provide public sector patients with alternative access to vital antiretroviral and other chronic medication, was implemented. This paper describes the protocol for a process evaluation of the programme compliance at the facility level in Namakwa district, to assess patient experiences and staff expectations of the programme; as well as, identifying factors that may affect the programme implementation so that guidance can be given on which approach to take to achieve programme objectives.

Methods and analysis A multimethod approach will be used in a cross-sectional process evaluation of the CCMDD programme at $11 \mathrm{PHC}$ facilities in Namakwa district. These methods will use checklists to assess programme compliance and subsequently gain an understanding of whether the programme was implemented as planned. Structured questionnaires together with focus group discussions will be conducted with selected patients enrolled in the programme and facility staff to determine patient experiences with and staff expectations of the programme, respectively. Furthermore, in-depth interviews will be conducted with key actors to explore barriers and facilitators of the programme implementation. Descriptive statistics will be conducted to analyse the quantitative data and an inductive interpretive approach will be used to analyse the qualitative data.

Ethics and dissemination The protocol was approved by Stellenbosch University Health Research Ethics Committee (S19/02/047) and the study will be conducted in line with the principles of the Declaration of Helsinki (1964). Findings from the study will be communicated to the study population, and at appropriate local and international conferences, in addition to publishing in peer-reviewed journals.
Strengths and limitations of this study

- A multimethod approach will give an in-depth understanding of multiple perspectives of the Central Chronic Medicines Dispensing and Distribution (CCMDD) programme.

- The results of this process evaluation may be used to make improvements in the implementation of CCMDD programme in the future.

- The study will determine patients' experiences with the CCMDD programme and staff knowledge and expectations of the programme.

- Factors affecting programme compliance will be determined through in-depth interviews with key informants.

- Findings from the study may not be generalisable to other districts in South Africa.

\section{INTRODUCTION}

The current population of South Africa (SA) is over 58 million $^{1}$; yet, its public health system covers as much as 45 million or almost $80 \%$ of the population. ${ }^{2}$ The quadruple disease burden, which includes tuberculosis $(6.5 \%-$ ranked first), HIV and AIDS (4.8\%—ranked fifth); high maternal, new born and child mortality; high levels of violence and injuries; as well as non-communicable diseases (NCDs), largely contributes towards the 10 leading causes of death in the country. ${ }^{3}$ NCDs are a common reason for patients seeking services at primary healthcare (PHC) facilities $^{4}$ and indicative of growing trends in death rates due to cardiovascular diseases. This includes ischaemic heart disease $(2.8 \%-$ ranked ninth) and other heart disease (5.1\% - ranked third); diabetes $(5.5 \%$ ranked second) resulting from high rates of obesity, ${ }^{3-6}$ hypertension $(4.4 \%$-ranked sixth) ${ }^{4}{ }^{5} 78$; and cerebrovascular diseases (5.1\%-ranked fourth). Unfortunately, 
SA does not have the infrastructure and resources to deal with the increasing chronic disease burden, which includes the change in categorisation of HIV/AIDS from acute to chronic ${ }^{9}$ due to treatment advances. ${ }^{10}$

The South African National Health Care Facilities Audit ${ }^{11}$ conducted in 2012 identified poor infrastructure; limited access to and availability of essential medicines; long-waiting times and poor service delivery as a national problem. However, the problem was found to be more predominant in the rural PHC facilities in the Northern Cape $(\mathrm{NC})$ province, which render nurse-driven primary healthcare (PHC) services. $^{11}{ }^{12}$ The epidemiological profile of SA together with a shortage and maldistribution of health professionals, especially in rural areas, ${ }^{13}$ are hindering the ability of supply chain systems to function optimally. This places an enormous strain on public healthcare facilities, specifically on its available resources,${ }^{14}$ resulting in poor quality of care; and subsequently, delaying the realisation of Universal Health Coverage or National Health Insurance. ${ }^{13}$

From a facility staff perspective, on average as much as $70 \%$ of the daily prescription load at PHC facilities are burdened with servicing repeat prescriptions, this immediately leads to congestion and unfavourable service provider-to-patient ratio. The latter contributes towards overburdened staff who do not have the capacity, and sufficient procurement practices, ${ }^{14}$ to adequately deal with unexpected pharmaceutical demand and supply requirements. This does not only contribute to medicine shortages, but also to inadequate counselling and education of patients due to time constraints. ${ }^{715}$ These challenges have also contributed towards poor self-efficacy for patients on chronic medications and ultimately suboptimal levels of adherence. ${ }^{16}$

Similarly, from a patient perspective, a typical stable chronic patient with a repeat prescription will be required to collect monthly repeats at a PHC facility between 6 -monthly clinical assessments. However, the experience of this patient at the facility is usually one of long waiting times and poor service delivery and often the patient is expected to return to the facility to collect medication, which was out of stock. This also poses potential adherence barriers that may lead to poor health outcomes ${ }^{17}$ and, in addition, place financial strain on the patient in terms of transport costs and loss of income. ${ }^{16}$

SA is often criticised for having health outcomes disproportionate to its spending, ${ }^{18}$ hence, the adoption of a small changes approach, defined as a process of changing or improving the current chronic medicines system. ${ }^{19}$ This approach is inexpensive and will have immediate effects rather than big changes approach such as changing infrastructure and employing more staff, which is more expensive and its effects only seen in the long term. ${ }^{19}$ The approach lead to the introduction of the Central Chronic Medicines Dispensing Distribution (CCMDD) programme ${ }^{20}$ on February 2014 by the South African National Department of Health $(\mathrm{NDoH})$ in eight of the nine provinces. The ninth province, the Western
Cape Province, has been running a similar programme, named the chronic dispensing unit (CDU), for over a decade. ${ }^{21}{ }^{22}$ While the CCMDD programme is described as 'a vehicle towards universal access to antiretroviral and other chronic medications in $\mathrm{SA}^{\prime},{ }^{20}$ it also alleviates pressure on PHC facilities. Currently the programme, together with other developments ${ }^{3}$ and the availability of list of medicines provided by $\mathrm{NDOH}$, which can be interchanged, ${ }^{23}$ provides improved and alternate access to certain chronic conditions for stable patients in the public sector.

The disparity in health services provided by both public and private sectors in SA, where the public sector receives $40 \%$ of health expenditure compared with $60 \%$ by the private sector, ${ }^{24}$ is widening the gap between the poor and the rich, adding to the urban/rural inequalities in medication accessibility and availability. ${ }^{25}$ Therefore, like any national policy, the CCMDD programme contributes towards strengthening the partnership between the public and private sectors. As a result, the mechanism of this programme is built on the use of a third party, from the private sector, to fill repeats and to distribute these filled repeats to various pick up points (PUP) away from public health facilities. These patientnominated PUPs can include private sector pharmacies, which typically offer longer trading hours, including weekends, than the public sector facilities. In addition, they are often more accessible from places of employment and, when coupled with shorter waiting times and minimal stock outs, allows for the collection of medicines without the need to take leave or lose income for a day. This is relevant as transport costs and loss of income, especially for those in rural areas, often prevent people from accessing healthcare.

The proposed study seeks to improve clinical outcomes among patients on chronic medication by assessing programme implementation so that interventions can be developed to improve quality and continuity of services. It seeks to evaluate the CCMDD programme compliance at facility level and determine if they are in accordance with the provincial guidelines, and determine patient experiences with and staff expectations of the programme in Namakwa district in SA. Furthermore, the study will identify factors that affect implementation of the programme so that guidance can be given on which approach to take to achieve the programme objectives. These will be achieved through the following objectives:

1. To assess the overall CCMDD programme compliance according to the NC provincial guidelines in Namakwa district.

2. To determine patient experiences with the programme in Namakwa district.

3. To determine whether the CCMDD programme met the expectations of facility staff in Namakwa district.

4. To investigate the barriers and facilitators associated with the implementation of the CCMDD programme in Namakwa district. 


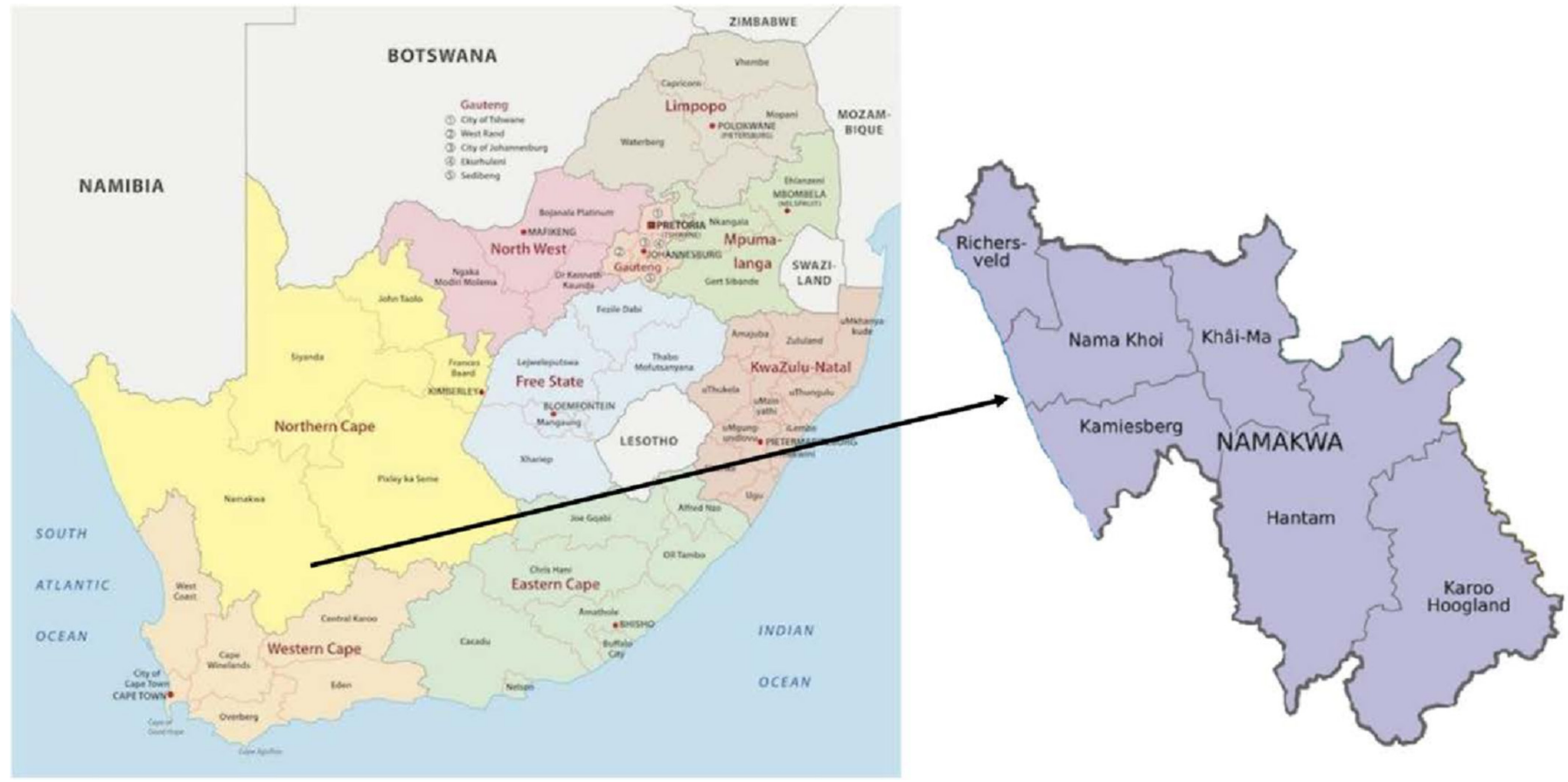

Figure 1 Map of South Africa showing the study settings (Namakwa district, Northern Cape Province).

\section{METHODS AND ANALYSIS \\ Design}

Multimethods approach involving both quantitative and qualitative methods will be used in a facility-based crosssectional process evaluation of the CCMDD programme, based on the enrolment rates found in the CCMDD Namakwa enrolment report for the period June 2017 to May 2018. The study design was selected because an in-depth understanding of multiple perspectives of the CCMDD programme was required to obtain the study objectives; and the study will commence in October 2019 until March 2020.

\section{Study setting}

The Namakwa district is situated in the western part of the NC, comprising of six subdistricts - Richtersveld, Nama Khoi, Khai Ma, Kamiesberg, Hantam and Karoo Hoogland (figure 1). It is the largest of the five districts in the $\mathrm{NC}$ province and covers a geographical area of approximately $126747.43 \mathrm{~km}^{2}$ with the smallest and most dispersed population of approximately 115, 842 people when compared with other districts. Most of the population in this rural district are predominantly of Afrikaans ethnicity $(83.90 \%)$ followed by White $(11.80 \%)$, Black African $(4.18 \%)$ and Indian/Asian $(0.12 \%)$. The poverty rate in the district was estimated to be $26.2 \%$ in 2014 .

There are currently 35 healthcare facilities in this district; however, access to these facilities is directly dependent on the spread of the facilities within a geographical space. ${ }^{26}$ Thus, due to the need for access to healthcare, better CCMDD enrolment rates were expected, since Namakwa was the last district in SA to implement the programme. The district could draw on experiences from other provinces and districts such as the Western Cape
Province, which has 13 years experience that has been translated into a facility manual and various procedures to aid implementation processes in the other provinces. ${ }^{16}$

\section{Facilities}

There are 19 health facilities, which have implemented the CCMDD programme in the district (table 1). Eleven of these facilities will be randomly selected from the district based on the highest and lowest enrolment rates found in the CCMDD Namakwa enrolment report for the period June 2017 to May 2018. However, all facilities in the Richtersveld (1), Kamiesberg (2) and Karoo Hoogland (2) subdistricts will be included in the study. Proportional sampling will be used to allocate CCMDD enrolments selected into the study per facility (table 1 ).

\section{Sampling and sampling techniques}

Data sources will be sampled from provincial, district and subdistrict and facility levels.

\section{Facility level}

Patient folders (objective 1)

\section{Sample size}

The sample size $(n=262)$ was derived by using the following parameters: $\mathrm{N}=(\mathrm{Z})^{2} \mathrm{P}(1 \mathrm{P}) / \mathrm{D}^{2}$, where ' $\mathrm{Z}$ ' is $95 \%$ confidence level), ' $\mathrm{P}$ ' is the expected prevalence $(70 \%)$, and ' $\mathrm{D}$ ' is the level of precision $(5 \%) .{ }^{27}$ The sample size was then adjusted to the finite population of 1385 enrolled CCMDD patients at the selected facilities as listed in the Namakwa district CCMDD enrolment report dated on $25 / 05 / 2018$ by using the formula: $S=n /[(1+n /$ population)]. 
Table 1 Sub-districts and health facilities in Namakwa district, Northern Cape (NC) Province

CCMDD

enrolments

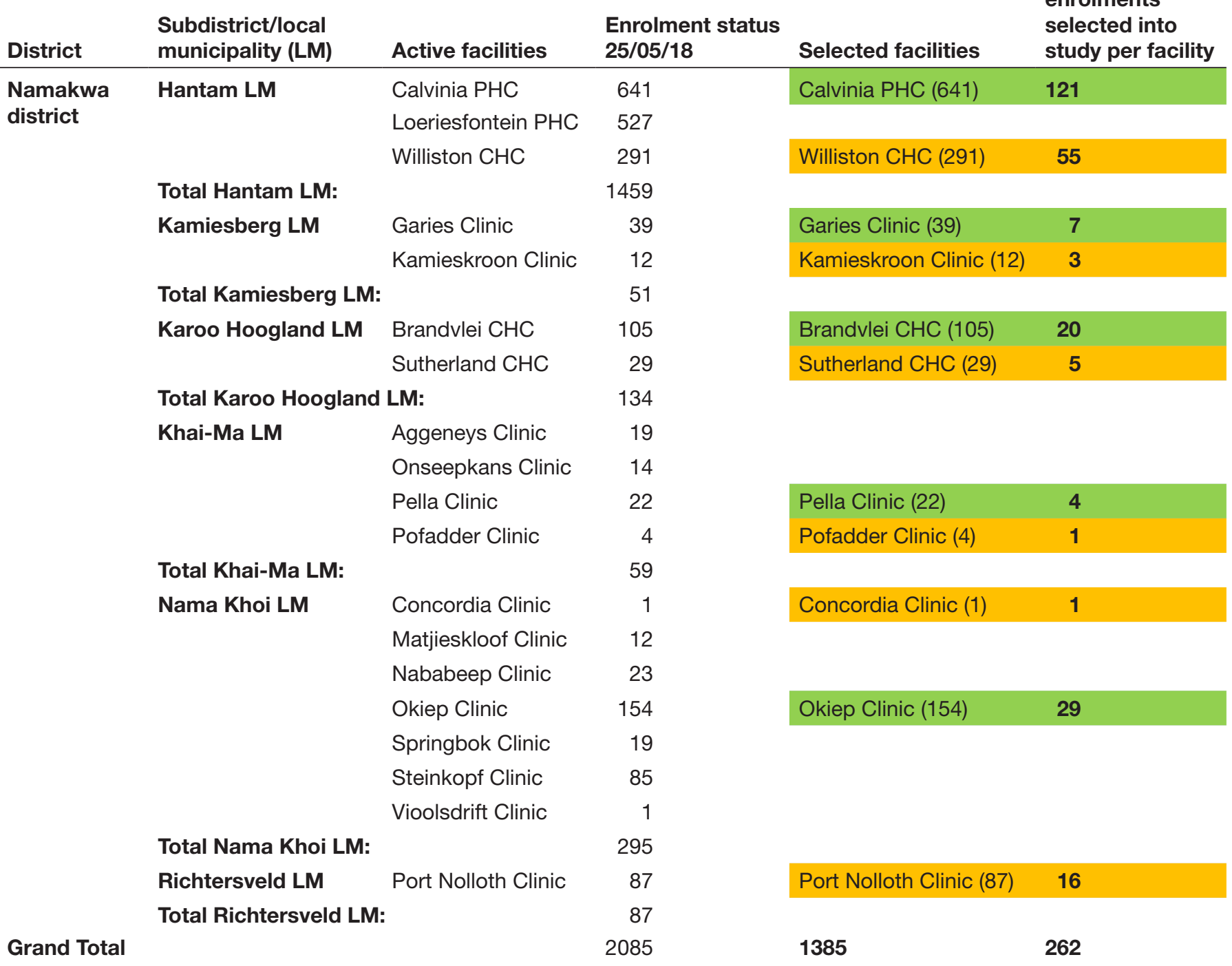

Green highlighted facilities=high enrolment rates.

Yellow highlighted facilities=low enrolment rates.

Source: Namakwa district CCMDD enrolment report-NC province operational data 2018.

CCMDD, Central Chronic Medicines Dispensing and Distribution; $\mathrm{CHC}$, community health centre; PHC, public healthcare; PHC, public healthcare.

A systematic sampling technique, with random starting point, will be used to select the required number of patient folders in each of the facilities.

\section{Patients (objective 2), and facility staff (objectives 1 and 3)}

Participants (patients and facility staff) will be randomly selected from each of the participating facilities; and will be contacted telephonically. Only chronic patients, and facility staff who are over the age of 18 years and who have given informed consent will be included in the study.

The required number of participants will be selected using a systematic sampling technique with random starting point. A purposive sample of 55 patients and 55 facility staff will be randomly selected from the programme register obtained from the CCMDD service providers at the facility level and staff register obtained from the district office, respectively (table 2). During this process, the research team will determine whether the selected patient or facility staff member met the inclusion criteria. If the inclusion criteria are not met, the reason for non-inclusion will be recorded and the research team moved on to the next name on the programme register or employee register in the direction in which the systematic sampling was done, until the total number of eligible patients and facility staff members will be sampled.

Provincial, district and subdistrict level

Key informants (objective 4)

Key informants will be purposefully selected based on their position in the provincial department of health 
Table 2 Overview of quantitative instruments and sample size by facility

\begin{tabular}{llrrrrrrrrrrrr}
\hline & Instrument & $\mathbf{1}$ & $\mathbf{2}$ & $\mathbf{3}$ & $\mathbf{4}$ & $\mathbf{5}$ & $\mathbf{6}$ & $\mathbf{7}$ & $\mathbf{8}$ & $\mathbf{9}$ & $\mathbf{1 0}$ & $\mathbf{1 1}$ & Total \\
\hline 1. & Checklist & 121 & 55 & 7 & 3 & 20 & 5 & 4 & 1 & 1 & 29 & 16 & 262 \\
2. & Structured questionnaires with patients & 5 & 5 & 5 & 5 & 5 & 5 & 5 & 5 & 5 & 5 & 5 & 55 \\
3. & Structured questionnaires with facility staff & 5 & 5 & 5 & 5 & 5 & 5 & 5 & 5 & 5 & 5 & 5 & 55 \\
& Total interviews per facility & 131 & 65 & 17 & 13 & 30 & 15 & 14 & 11 & 11 & 39 & 26 & 372 \\
\hline
\end{tabular}

1: Calvinia PHC 2: Williston CHC 3: Garies PHC 4: Kamieskroon PHC 5: Brandvlei 6: Sutherland 7: Pella PHC 8: Pofadder PHC 9: Concordia PHC 10: Okiep PHC 11: Port Nolloth PHC.

$\mathrm{CHC}$, community health centre; $\mathrm{PHC}$, public healthcare.

and experience with the implementation of the CCMDD programme, as defined in the CCMDD facility manual. ${ }^{16}$ They will include provincial programme manager, district manager, district pharmaceutical manager, health Area Managers (HAMs) and sub-district pharmacists. Participants will be contacted telephonically. Only key informants who have given informed consent will be included in the study.

\section{Instrument design}

The instruments (online supplementary annexure A-F) developed through the guidance of information provided from CCMDD policies and guidelines ${ }^{16}$ will be used. Information sought will include the actual programme practices (online supplementary annexure A), programme experiences of patients (online supplementary annexure C,D), programme expectations of nurses (online supplementary annexure $\mathrm{C}, \mathrm{E})$, as well as, the factors that affect the implementation of the programme (online supplementary annexure F). Where necessary, the instruments will be revised using comments derived from the pilot.

\section{Pilot testing}

The main purpose of the pilot is to test the suitability of the research instruments and identify whether further adjustments are needed. Draft instruments will be administered to random selected CCMDD programme participants at Port Nolloth community health centre. The pilot will include five patient folder reviews; structured questionnaires with two patients and two facility staff; two focus group discussion (FGDs) with 6-8 patients and 3-6 facility staff members, respectively; interviews with one key informants involved implementation of the programme in the district. The pilot phase will involve reviewing chronic patient folders, and asking participants questions pertaining to their programme experiences (patients) and expectations (facility staff). It will include interviews with key informants to determine programme facilitators and barriers, which will help to answer study objectives and assist the researcher in approximating the time needed to complete the questionnaire for the actual study as shortage of staff and service delivery, remain an issue of concern in this study.

\section{Data collection}

Quantitative and qualitative data collection will take place simultaneously. Table 3 illustrates the linkages between study objectives, data collection methods, instruments and data sources.

Quantitative data related to information requirements will be collected through:

a. Checklists (online supplementary annexure A) will be used to assess programme compliance (objective 1) from patient folders

- To ascertain whether the correct programme processes have been followed (objective 1) the researcher will review the required number of chronic patient folders at the facilities sampled into the study. The duration of the assessment will be determined during the pilot and the time of the assessment will be determined on the availability of the facility that is, operational hours. However, we are hoping this will take between 10 to 20 min per folder.

b. Structured questionnaires (online supplementary annexure $\mathrm{B}, \mathrm{C}$ ) will be used to obtain data from a purposive sample of 55 patients and 55 facility staff to understand patients' experience with the CCMDD programme (objective 2) and facility staff's expectations of the CCMDD programme. In addition, the questionnaires will be used to collect data around programme compliance practices and will therefore be used to complement the checklist (online supplementary annexure A) in answering the first objective.

Qualitative data related to information requirements will be collected through:

a. Like the structured questionnaires, the FGDs with patients and facility staff will be used to determine patients' experience with the CCMDD programme (objective 2) and facility staff's expectations of the CCMDD programme (objective 3 ).

- Two FGDs will be conducted with approximately 6-8 patients and two FGDs with approximately 3-6 facility staff from the facility with the highest programme enrolments, that is, Calvinia PHC and the facility with the lowest programme enrolments, that is, Concordia PHC in the district, respectively.

- The patients and facility staff will be randomly identified for the interviews, which will last between 45 min to 1 hour. The FGDs will be conducted using 
Table 3 Linkages between study objectives, data collection methods, instruments and data sources

\begin{tabular}{|c|c|c|c|c|}
\hline Data collection & $\begin{array}{l}\text { Objective } 1 \\
\text { (programme } \\
\text { compliance } \\
\text { assessment) }\end{array}$ & $\begin{array}{l}\text { Objective } 2 \\
\text { (determination of } \\
\text { patient experience } \\
\text { with programme) }\end{array}$ & $\begin{array}{l}\text { Objective } 3 \\
\text { (determination } \\
\text { of facility staff } \\
\text { expectations with } \\
\text { programme) }\end{array}$ & $\begin{array}{l}\text { Objective } 4 \\
\text { (barriers and facilitators } \\
\text { associated with } \\
\text { programme compliance) }\end{array}$ \\
\hline \multicolumn{5}{|l|}{ Quantitative methods } \\
\hline $\begin{array}{l}\text { Checklists of patient folders (online } \\
\text { supplementary annexure A) }\end{array}$ & $x$ & & & \\
\hline $\begin{array}{l}\text { Structured questionnaires with facility staff } \\
\text { (online supplementary annexure C) }\end{array}$ & $x$ & & $\mathrm{x}$ & \\
\hline \multicolumn{5}{|l|}{ Qualitative methods } \\
\hline $\begin{array}{l}\text { Focus groups with patients (online } \\
\text { supplementary annexure D) }\end{array}$ & & $x$ & & \\
\hline $\begin{array}{l}\text { In-depth interviews with key informants } \\
\text { (online supplementary annexure E) }\end{array}$ & & & & $\mathrm{X}$ \\
\hline \multicolumn{5}{|l|}{ Data sources } \\
\hline Patient folders & $x$ & & & \\
\hline $\begin{array}{l}\text { Chronic patients enrolled into the } \\
\text { programme }\end{array}$ & & $x$ & & \\
\hline Facility staff & $x$ & & $\mathrm{x}$ & \\
\hline Multiple key actors & & & & $X$ \\
\hline
\end{tabular}

an interview guide (online supplementary annexure $\mathrm{D}, \mathrm{E})$.

- Interviews will be conducted by experienced and trained research team members at the PHC facility; and the interview will be recorded with a voice recorder if permission was granted by the interviewee. b. In-depth interviews (IDIs) with key informants (ie, provincial programme manager, district manager, district pharmaceutical manager, HAMs, district clinical specialist and subpharmacists) will be undertaken to explore barriers or programme facilitators during programme implementation in the district (objective 4).

Table 4 Overview of categories and number of participants and key informants interviewed by organisational levels (ie, provincial, district, subdistrict and facility)

\begin{tabular}{|c|c|c|c|c|c|c|}
\hline Category of participant & $\begin{array}{l}\text { Provincial- } \\
\text { level actors }\end{array}$ & $\begin{array}{l}\text { District-level } \\
\text { actors }\end{array}$ & $\begin{array}{l}\text { Subdistrict- } \\
\text { level actors }\end{array}$ & $\begin{array}{l}\text { Facility 1: } \\
\text { calvinia } \\
\text { (highest } \\
\text { enrolment rates) }\end{array}$ & $\begin{array}{l}\text { Facility 9: } \\
\text { Concordia } \\
\text { (lowest } \\
\text { enrolment rates) }\end{array}$ & Total \\
\hline Provincial programme manager (1) & 1 & & & & & 12 \\
\hline District manager (1) & & 1 & & & & \\
\hline District pharmaceutical manager (1) & & 1 & & & & \\
\hline Health area managers (4) & & & 4 & & & \\
\hline Subdistrict pharmacists (4) & & & 4 & & & \\
\hline \multicolumn{7}{|c|}{ FGDs with facility-level participants $( \pm 6-8)$ patients and $( \pm 3-6)$ facility staff at each respective FGD } \\
\hline Patients $(n=2)$ & & & & 1 & 1 & 4 \\
\hline
\end{tabular}

FGDs, focus group discussions; IDIs, in-depth interviews. 
- IDIs will be conducted with approximately 12 key informants across the province (table 4).

- The interviews, which will last between $45 \mathrm{~min}$ and 1 hour, will be conducted by experienced and trained researchers using an interview guide (online supplementary annexure F). The interview will be recorded with a voice recorder if permission was granted by the interviewee.

- Provincial informants will be interviewed telephonically; however, these interviews will follow the same procedures as the face-to-face interviews.

\section{Data analysis}

Analysis of quantitative data (objectives 1, 2, 3)

Data extracted from the checklist and questionnaires will be captured directly into the Research Electronic Data Capture software and exported into STATA V.14 for analysis. To ensure data quality assurance, the lead author will spot check a few questionnaires, including the checklists, against entered data for missing values and logical inconsistencies. However, no reinterviewing of participants will be possible as all data are anonymous. All the data collected with the checklist and structured questionnaire will be described with means, SD and percentages, if normally distributed or medians and IQR, if nonnormally distributed (skewed) data. Categorical data will be summarised using proportions, frequencies and $95 \%$ CIs. Data will also be represented graphically using bar charts and histograms. This descriptive statistics included demographic variables, that is, age and socioeconomic variables, that is, highest level of education which will be collected to identify if these independent variables had an effect on the dependent variables or main outcome variables.

Data from the checklist and structured questionnaires will be analysed to describe the implementation practices of the CCMDD programme and patients' experiences with and facility staff's expectations of the programme, respectively. Of particular interest will be the analysis of any differences in implementation practices between different facilities and differences in patients' experiences and facility staff's expectations across different facilities in the district.

\section{Analysis of qualitative data (objective 2-4)}

Audio recording from patients, facility staff and key informant information gathered through the IDIs and FGDs will be transcribed and translated to English by an experienced transcriber; a general inductive approach based on the techniques of systematically identifying emerging themes, categories or patterns from the data will be used. ${ }^{27}$ Responses from participants will be independently coded by the lead author and an experienced qualitative analyst. Preliminary analyses will be undertaken by examining the transcripts, which will be read over and over, until similar emerging themes will be identified, coded and categorised. These independent analyses will then be compared for consistency; areas of discrepancies will be identified through critical evaluation of the sets of themes. The source quotes will then reviewed and agreed on, and a final thematic report will be generated from the combined analyses. The results will be shared with managers and staff at a district level for validation depending on the content.

\section{Patient and public involvement}

The research questions and study design were developed from experience through daily interactions and engagement with patients and the CCMDD programme at the facility level. In addition, the multimethods study design will involve patients through their participation in piloting the structured questionnaires and FGDs. However, no patient will be involved in recruiting participants and conducting the study.

\section{Ethics and data dissemination \\ Ethics}

This research will be conducted in accordance with the ethical code of conduct as described in the Declaration of Helsinki and the South African Good Clinical Practice Guidelines as well as the Ethical Guidelines for Research as outlined by the South African Medicine Research Council.

\section{Informed consent}

The facility staff and patients will be informed of the purpose of the study in person prior to being invited to participate in the study. If they agree to do so, the informed consent document will be issued to them in their language of choice (Afrikaans or English). This is done to ensure that all respondents understand their rights, the study procedures that will be followed, measures that will be taken to protect the patients and their anonymity and the potential risks and benefits of participating in the study. Written informed consent will then be obtained and a copy of the document, if requested, will be provided to the respondent for their personal record. The research team will objectively assist any illiterate respondents enrolled into the study.

\section{Confidentiality}

The completed checklists, structured questionnaires and recorded interviews will be safely stored in a secured password access-controlled computer, only accessed by the authors. Any personal identifiers linked to the research participants will be removed.

\section{Reimbursement}

All participants (ie, patients, facility staff and key informants) will be compensated for their time; in addition, patients will be reimbursed for any out of pocket expenditure incurred as a result of participating in the study.

\section{Beneficence and non-maleficence}

It is envisaged that no harm will be incurred as a result of participation, since no intervention will be done. The participants will not benefit from the study and 
the outcomes of the study will provide insight into the implementation of the programme and inform decision making.

\section{Data dissemination}

The results of the study will be published in a peerreviewed journal; preferably, the same journal in which the protocol will be published (BMJ). The authors will be available to explain the study findings to any interested party, and will make every attempt to have these results included in any decision making or healthcare intervention on a local, provincial and national level. Thus, study findings will be presented at appropriate local and international conferences and any other health promotion audiences as opportunity presents. This will be in addition to results being disseminated to the study participants and population with the help of community health workers.

\section{DISCUSSION}

Despite the importance of the CCMDD programme as a platform for universal access to antiretroviral and other chronic medications in $\mathrm{SA},{ }^{21}$ the programme enrolment rate in the Namakwa district (NC) is far from optimal. Medicines supplies, patient enrolment processes, healthcare professionals' compliance to legislation and policies, management of non-collected medicines (emanating from patients' missed appointments) have all been cited as factors central to the functioning of the $\mathrm{CDU}$, a similar programme in the Western Cape. ${ }^{20}$

The proposed protocol seeks to evaluate the CCMDD programme compliance at facility level and determine if they are in accordance to provincial guidelines, and determine patient experiences with and staff expectations of the programme in Namakwa district in SA. Furthermore, it hopes to give an overview of the factors that play a part in the implementation of the CCMDD programme in the Namakwa district. This will be achieved by identifying factors that affect implementation of the programme through the analysis of any differences in implementation practices between different facilities and differences in patients' experiences and facility staff's expectations across different facilities in the district; so that guidance can be given on which approach to take so that programme objectives can be achieved.

It will also offer an understanding of the drivers of this process, with an emphasis on targeted interventions to promote implementation of the programme so that programme objectives can be achieved.

The proposed cross-sectional process evaluation uses a multimethods approach, which will provide an in-depth understanding of multiple perspectives of the CCMDD programme. This will be obtained through the involvement of a wide range of stakeholders, at various levels of programme implementation, so that rich data around programme implementation can be obtained. The process evaluation will aid in determining whether the programme was implemented as planned, as well as, identifying the causes of poor programme implementation and the degree of acceptability of the programme by the facility staff and patients in the Namakwa district. ${ }^{28}$

The outcome of this study hopes to identify factors that affect programme compliance and the possible implications thereof. The Western Cape study similarly identified missed appointments to affect $8 \%-12 \%$ of patients each month, which results in cost to government for services rendered by contractor, potential losses due to expired medicines, additional workload for the contractor and health facility staff and potential negative therapeutic outcomes for patients. ${ }^{21}$ Possible factors might be identified through the knowledge test, which forms part of the staff questionnaire, and will give insight into healthcare professionals' compliance to legislation and policies and patient enrolment processes, as well as, the implications thereof, which might be a result of gaps between knowledge and practice. A concept like this is not foreign, and has been extensively reported in the implementation of other health programme like Prevention of mother-tochild transmission of HIV (PMTCT), and is addressed through training.

A major limitation of this study may be the inability to generalise the findings to other districts, since the study will be conducted in one of the 52 districts in SA, which may not have the same characteristics as other districts and may not necessarily reflect the programme performance in these districts. However, the findings can help CCMDD programme managers at provincial, district and subdistrict levels develop targeted interventions to improve programme performance in the Namakwa district.

Contributors KGS and EN conceived the study. KGS drafted and revised the manuscript, while EN provided methodological support and critical conceptualisation and contribution towards developing and refining the manuscript. All authors read and approved the final manuscript.

Funding This study will be funded by the South African Medical Research Council.

Map disclaimer The depiction of boundaries on this map does not imply the expression of any opinion whatsoever on the part of BMJ (or any member of its group) concerning the legal status of any country, territory, jurisdiction or area or of its authorities. This map is provided without any warranty of any kind, either express or implied.

Competing interests None declared.

Patient consent for publication Not required.

Ethics approval The protocol has been approved by Stellenbosch University Health Research Ethics Committee (S19/02/047) and study permission has been obtained from the Northern Cape Department of Health (NAM_201901_001).

Provenance and peer review Not commissioned; externally peer reviewed.

Open access This is an open access article distributed in accordance with the Creative Commons Attribution Non Commercial (CC BY-NC 4.0) license, which permits others to distribute, remix, adapt, build upon this work noncommercially, and license their derivative works on different terms, provided the original work is properly cited, appropriate credit is given, any changes made indicated, and the use is non-commercial. See: http://creativecommons.org/ licenses/by-nc/4.0/

ORCID iD

Edward Nicol http://orcid.org/0000-0001-6019-9259 


\section{REFERENCES}

1 Worldometers. Available: https://wwww.worldometers.info/worldpopulation/south-africa-population/ [Accessed 5 Oct 2019].

2 Statistics South Africa. Department of statistics South Africa. public healthcare: how much per person? Available: www.statssa.gov.sa [Accessed 5 Oct 2019].

3 Statistics South Africa. Mortality and causes of death in South Africa, findings from death notification, 2016. Available: http://www.statssa. gov.za/publications/P03093/P030932016.pdf [Accessed 5 Oct 2019].

4 Meyer JC, Schellack N, Stokes J, et al. Ongoing initiatives to improve the quality and efficiency of medicine use within the public healthcare system in South Africa; a preliminary study. Front Pharmacol 2017;8:751.

5 Mash B, Fairall L, Adejayan O, et al. A morbidity survey of South African primary care. PLoS One 2012;7:e32358.

6 Stokes A, Berry KM, Mchiza Z, et al. Prevalence and unmet need for diabetes care across the care continuum in a national sample of South African adults: evidence from the SANHANES-1, 2011-2012. PLoS One 2017;12:e0184264

7 Basu S, Wagner RG, Sewpaul R, et al. Implications of scaling up cardiovascular disease treatment in South Africa: a microsimulation and cost-effectiveness analysis. Lancet Glob Health 2019;7:e270-80.

8 Rampamba EM, Meyer JC, Godman B, et al. Evaluation of antihypertensive adherence and its determinants at primary healthcare facilities in rural South Africa. J Comp Eff Res 2018;7:661-72.

9 Deeks SG, Lewin SR, Havlir DV. The end of AIDS: HIV infection as a chronic disease. The Lancet 2013;382:1525-33.

10 The conversation. How HIV became a treatable, chronic disease, 2015. Available: https://the conversation.com/how-hiv-became-atreatable-chronic-disease-51238\#comments-container [Accessed 5 Oct 2019].

11 Department of Health. The National health care facilities baseline audit: national summary report, 2013. Available: http://www.hst. org.za/sites/default/files/NHFA_webready_0.pdf [Accessed 17 Aug 2017].

12 Massyn N, Peer N, Padarath A, et al. District health barometer 2015/16. health systems trust, 2016. Available: https://www.hst.org. za/publications/Pages-District-Health [Accessed 5 Oct 2019].

13 IOL. Health shortages make NHI a 'pipe dream', 2018. Available: https://www.iol.co.za/news/south-africa/health-staff-shortagesmake-nhi-a-pipe-dream-18480717 [Accessed 5 Oct 2019].

14 Magadzire BP, Ward K, Leng HMJ, et al. Inefficient procurement processes undermine access to medicines in the Western Cape Province of South Africa. S Afr Med J 2017;107:581-4.
15 Moosa A, Bezuidenhout S, Meyer JC, et al. Knowledge regarding medicines management of type 2 diabetes amongst patients attending a community health centre in South Africa. J Pharm Health Serv Res 2019:10:13-28.

16 Support Partner: The Health Systems Trust. Central chronic medicines dispensing and distribution (CCMDD) facility manual, 2015. Available: https://www.hst.org.za/hstconference/ hstconference2016/Presentations/roma_ramphal_hst_conf_final_04. 05.2016.pdf

17 Vagiri RV. Relationship between adherence and health-related quality of life among HIV-patients in South Africa: findings and implications. J AIDS HIV Res 2018;10:121-32.

18 Kleinert S, Horton R. South Africa's health: departing for a better future? The Lancet 2009;374:759-60.

19 Du Toit J, Dames S, Boshoff R. Centralised dispensing - an affordable solution. South African Pharm J 2008:18-21.

20 Zeeman H. CCMDD: a vehicle towards universal access to anti Retrovirals and other chronic medicines in South Africa, 2017. Available: https://www.hst.org.za/hstconference/hstconference2016/ Presemtations/hst_conf_h_zeeman_28_04_2016.pdf [Accessed 5 Oct 2019].

21 Magadzire BP, Marchal B, Ward K. Improving access to medicines through centralised dispensing in the public sector: a case study of the chronic dispensing unit in the Western Cape Province, South Africa. BMC Health Serv Res 2015;15:513.

22 Du Plessis J. The chronic dispensing unit. South African Pharm J 2008:46-7.

23 National Department of Health. Policy for classifying medicines into therapeutic classes for purposes of therapeutic interchange, 2017. Available: https://www.gov.za/index.php/national-essential-medicinelist-committee-nemlc?download=3307:therapeutic-interchangepolicy-final [Accessed 5 Oct 2019].

24 National Department of Health. A policy on quality in health care for South Africa, 2007. Available: https://www.gov.za/sites/default/files/ gcis_document/201409/qhc-policy.pdf [Accessed 5 Oct 2019].

25 Harris B, Goudge J, Ataguba JE, et al. Inequities in access to health care in South Africa. J Public Health Policy 2011;32:S102-23.

26 National Department of Health. Namakwa district health plan 2018/2019-2020/2021. Northern Cape. Available: http://www.health gov.za/DHP/docs/DHP2018-21/Northern_Cape/Namakwa.pdf [Accessed 5 Oct 2019].

27 Verhoef $\mathrm{M} \mathrm{H}$. Writing an effective research proposal, 2004. Available: http://www.ais.up.ac.za/health/blocks/block2/researchproposal.pdf [Accessed 21 Sep 2018].

28 Royse D, Bruce AT, Deborah KP, et al. Program evaluation: an introduction. 4th edn. Thomson Brooks/Cole, 2006. 